\title{
Application of MM40 Invert in Direct Torque Control of PHEV
}

\author{
Zhixue Tong \& Baozhen Zhang \\ Xi'an University of Architecture and Technology, Xi'an 710055, China
}

\begin{abstract}
In order to meet the problem of motor torque control for plug in hybrid electric vehicle, direct torque control system is designed with Profibus-DP bus and Ethernet communication by using Siemens S7300 series PLC and MM 440. Structure and control scheme of the system is described. Hardware design of the system is given. Software design of the system is also completed. Based on the PC witch equipped with WinCC-Flexiblel, Human Machine Interface configuration of the system is made, which achieved humanmachine information exchange. Finally, the torque monitoring interface is given after the system is debugged successfully, which confirmed the feasibility of the design.
\end{abstract}

KEYWORD: Programmable logic controller (PLC); Human machine interface (HMI); MM440 inverter; Profibus-DP bus; Torque control; PHEV

\section{INTRODUCTION}

Currently in the energy management strategies of plug in hybrid electric vehicle substantially is essentially based on power or torque aspects control. Torque control is relatively simple, when in the state of switching process for driving hybrid the speed changes affected cannot be considered during the allocation of the engine and motor power, only on the motor torque control (Y.L.Han, 2013) (C.H.Wei et al, 2012). For this many articles put forward new ideas and methods from a different perspective, especially with the introduction of a variety of control theory, the new control strategy is continually appear. But how to test to verify control motor torque in hybrid is a problem in front of people.

Siemens production MicroMaster440 (referred MM440) inverter use high-performance vector control technology, provide low speed high torque output and excellent dynamic characteristics, with superior overload, and also achieve master / slave control and torque control (B.J.Pan, 2007), While with innovative BICO (internal function Internet) function it has unmatched flexibility (L.F.Fu et al, 2011).

This article will be established PROFIBUS-DP communication S7-300 and MM440 inverter, use the PC to operate the drive via Industrial Ethernet, thus controlling the drive reads and operating parameters to achieve the motor torque control.

\section{SYSTEM COMPONENTS}

The experimental setup consists of three parts: the monitoring unit, a control unit and a drive unit.

Monitoring unit consists of a PC equipped with WinCC-Flexible machine components.

The control unit consists of S7-300 PLC.

Drive unit consists of MM440 inverter and the motor.

MM440 inverter motor control via PROFIBUSDP communication module connected to the S7-300 CPU's DP interface. The PC provided with WinCC Flexible configuration software is connected via Ethernet to S7-300 PLC's CP-341 Ethernet module. By operating the PC will transmit the torque set point to S7-300 PLC, PLC according to the results of internal logic operation and PID operation modified drive control word, and fed the actual parameters value of system operation back to PC, thus achieving an entire communications network. The schematic of framework is shown in Fig.1. 
Industrial Ethernet

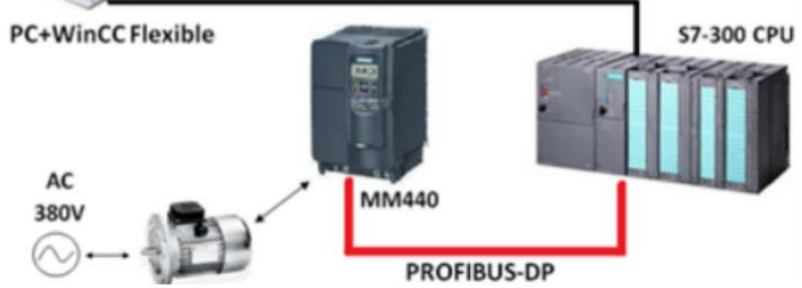

Fig 1 The composition of electric control system for Lab

\section{SYSTEM HARDWARE DESIGN}

Based on the needs of the system, selected Siemens 300 series PLC as the system controller, with integrated DP port for the compact 313C-2DP CPU. Using the $\mathrm{PC}$ as the user interface, and use general inverter Siemens MM440 to achieve AC motor torque control.

MM440 inverter as DP bus slave station, use the communication of Profibus-DP bus communication between the main stations S7-300. The PC equipped with WinCC Flexible is connected via Ethernet cable to the S7-300 PLC's CP-341 Ethernet module.

\subsection{DP communication S7-300 and MM440 inverter.}

MM440 using PROFIBUS-DP bus to connect to S7300 witch using PROFIBUS-DP protocol. ProfibusDP is one of the most successful field bus, has been widely used. DP master send request message (containing control and configuration information) to the DP slave continuously, DP slave is receiving messages, resolving address and comparing with its own station address. If it is sent to their own information, it send a response message (containing state information, etc.) Within a specified time. Meanwhile MM440 inverter also monitors the communication, if communication fails, MM440 drive will trigger an outdated response, notify the master station retransmits the message (X.H.Huang et al, 2012) (Z.W.Shi, 2013). MM440 inverter through communication panel (CB) to achieve this functionality.

When you select Profibus-DP communication, to make the inverter to work properly, it need to operate the control panel to set parameters closely related to DP communication. The main parameters are provided:

P700 is set to 6, allows the COM link communication board (CB) for inverter control;

$\mathrm{P} 1500$ is set to 6 , allows the COM link communication board (CB) for sending of the main set point;

$\mathrm{P} 1300$ is set to 22, sets specify inverter using vector-torque control mode without sensor feedback;

P0918 is set to 3, sets PROFIBUS address of the inverter;
P0927 set to 15 , enables DP interface changing parameters.

Moreover, while control of the inverter is used with the Siemens converter unique BICO features. BICO is a setting method associated with input and output functions of the inverter, Users can easily to define flexible drive IO port according to the actual process requirements (B.Fu et al, 2015). MM440 built BICO technology, the main settings are as follows:

P0719 is set to 0 , commands and set values are using BICO;

P2051.0 set to r0052, allow the status word 1 through $\mathrm{CB}$ board to send to the inverter.

\subsection{PC machine via Ethernet communication with S7-300 (J.M.Yin et al, 2008).}

With the development of computer science applications, Ethernet technologies have been gradually applied in automation and measurement \& control industry, and developed into a technology trend.

PC machine via Ethernet communications with S7-300 is based on TCP / IP communications protocols, with the use of Ethernet technology for data transmission.

\section{SOFTWARE DESIGN}

When using Siemens S7-300PLC programming software Step7 for system software design, it is divided into two processes: hardware configuration, communication procedures.

\subsection{Hardware Configuration.}

Hardware configuration is one indispensable step for Step7 software programming. Hardware configuration is installing the CPU, power supply, signal modules, communication processors and function modules and other equipment in strict accordance with the product version serial number to the corresponding rack, while the parameters of PLC hardware modules also to be set and modified. Direct Torque Control System hardware configuration is shown in Figure 2, wherein the attribute selection of MICROMASTER4 packet type is PPO1. 


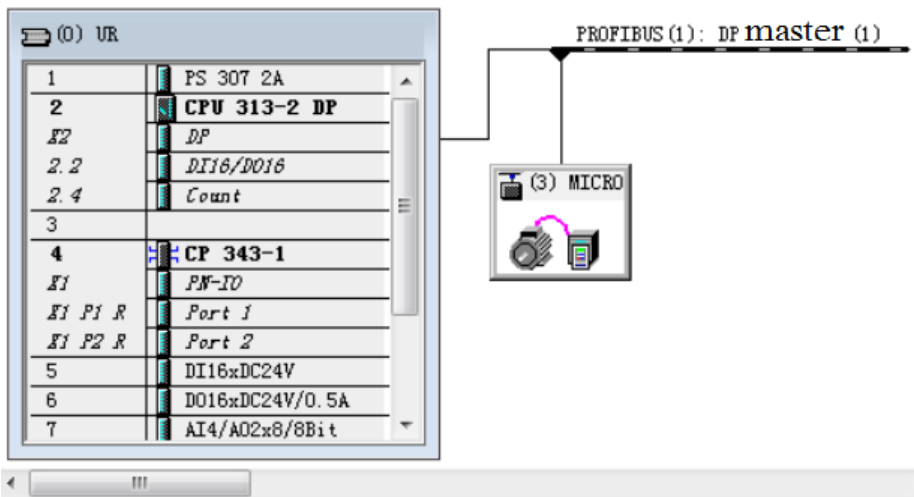

$\Rightarrow$ (3) MICROMASTER 4

\begin{tabular}{|c|c|c|c|c|c|}
\hline & DP ID & $\ldots$ & Order No. / Flag & I & $Q$ \\
\hline 1 & $4 A X$ & & 4 PKW, 2 PZD $\quad(\mathrm{PPO} 1)$ & $272 \ldots 279$ & $272 \ldots 279$ \\
\hline 2 & $2 A I$ & & $\mid \rightarrow 4$ PSR, & $280 \ldots 283$ & $280 \ldots 283$ \\
\hline
\end{tabular}

Fig 2 System hardware configuration figure

\subsection{Communication program (S.l.Yang, 2011).}

When using Profibus-DP communication mode, it first need to establish a data block DB1while the data in the data block address have to correspond with the slave MM440 PZD, PKW data area, then call the special function blocks SFC14 ("DPRD_DAT") and SFC15 ("DPWR_DAT") in the program, in order to achieve the read and write operations from master station S7-300 to slave station MM440 S7-300 data.

Part of the system program calls as follows:

CALL "DPWR_DAT"

LADDR: $=\mathrm{W} \# 16 \# 118$

//the starting address PZD of the hardware configuration

RECORD: =P\#DB1.DBX20.0 BYTE 4

//the data address corresponding to PZD data area

RET_VAL:=MW8

//Status Word of program block

CALL "DPRD_DAT"

LADDR: =W\#16\#118

RET_VAL:=MW6

RECORD: =P\#DB1.DBX8.0 BYTE 4

\section{DESIGN OF SYSTEMS' HMI (C.C.LIAO, 2011)}

HMI is a bridge to achieve two-way communication between the operator and the PLC. The system is created an interface by PC equipped with configuration software WinCC-Flexible, and achieve a communication both operational interface and PLC equipment via Ethernet.

\section{SYSTEM DEBUGGING AND RUNNING}

System debugging is checking the system whether or not to achieve the drive motor torque control under PROFIBUS-DP communication, and human- machine interface is correctly implement monitoring. It is available through online debugging function by opening Step 7, and perform the appropriate action on the system interface, to observe changes in the corresponding variables. So as to verify the design is correct and reasonable. Real-time monitoring interface of the torque value under normal operating conditions after the system debugged successfully is shown in Figure 3.

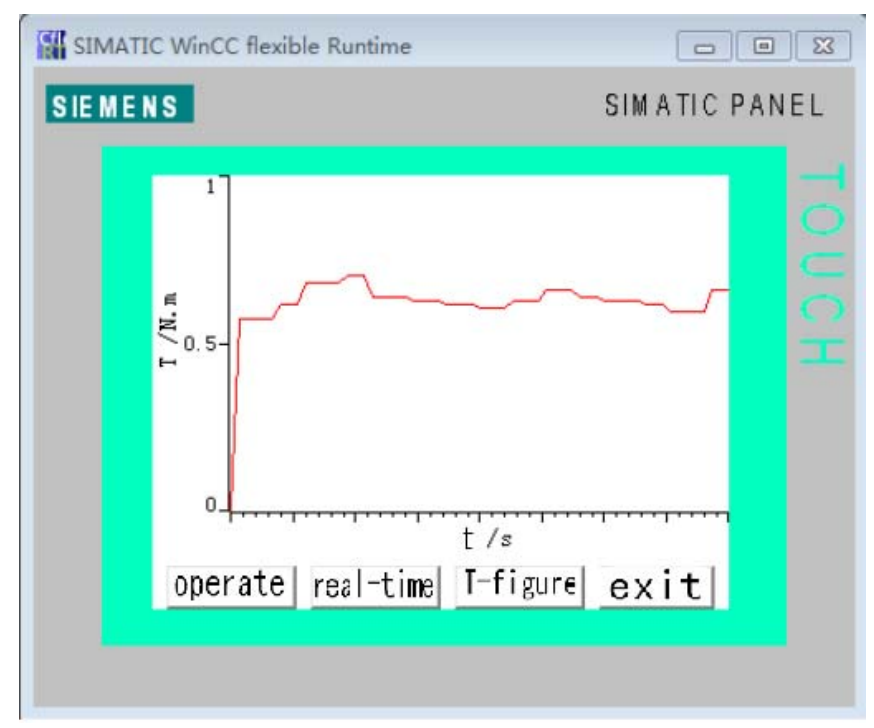

Fig 3 Real-time monitoring torque value

\section{CONCLUSION}

This paper built a system to verify motor torque control through Siemens S7-300 series PLC, MM440 inverter, PC machine equipped with WinCCFlexible. The system uses the Profibus-DP, Ethernet two kinds of typical communication. The paper gives specific hardware design and software design, and display system status conveniently through the man-machine interface programming configuration, set parameters and operation. The system both has real-time performance and high reliability characteristics, can be applied to hybrid test bench completely.

\section{REFERENCES}

B.Fu, X.D.Li, H.Z.Li: Electric Engineering, (2015) no. 4, p.4748.

B.J.Pan, Electric Machines \& Control Application, Vol.34 (2007) no. 8, p. 33-35.

C.C.Liao: SIEMENS Human Machine Interface Configuration and Application Technology, (2011) no. 2, p. 156-174.

C.H.Wei, F.Qiu, L.j.Chen, et al: Agricultural Equipment \& Vehicle Engineering, Vol.50 (2012) no.10, p. 1-5.

J.M.Yin, W.S.Wei, Y.Shi,et al: Computers and Applied Chemistry, (2008)no. 1, p. 25-26.

L.F.Fu, X.Zhang: BIAN PIN JI SHU, (2011) no. 1, p. 129-134. 
S.l.Yang: Zhongguo Baozhuang Keji Bolan, (2011) no. 9, p.910.

X.H.Huang, J.Cao, X.C.Wang: Computer Engineering and Design, Vol.33 (2012) no.6, p. 2467.

Y.L.Han: The Research of Dynamic Control during Modeswitches for PHEV (MS., Yanshan University, China 2013).

Z.W.Shi, Electronics World, (2013) no. 24, p.128-130. 